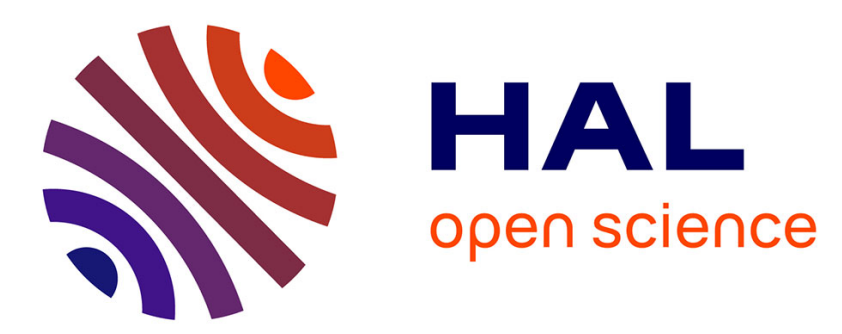

\title{
New image reconstruction algorithm for fluorescence optical tomography based on the adjoint radiative transfer equation
}

\author{
Fatmir Asllanaj, Ahmad Addoum
}

\section{- To cite this version:}

Fatmir Asllanaj, Ahmad Addoum. New image reconstruction algorithm for fluorescence optical tomography based on the adjoint radiative transfer equation. Optical Tomography and Spectroscopy of Tissue XIII, Feb 2019, San Francisco, France. pp.31, 10.1117/12.2507420 hal-03522812

\section{HAL Id: hal-03522812 \\ https://hal.science/hal-03522812}

Submitted on 12 Jan 2022

HAL is a multi-disciplinary open access archive for the deposit and dissemination of scientific research documents, whether they are published or not. The documents may come from teaching and research institutions in France or abroad, or from public or private research centers.
L'archive ouverte pluridisciplinaire HAL, est destinée au dépôt et à la diffusion de documents scientifiques de niveau recherche, publiés ou non, émanant des établissements d'enseignement et de recherche français ou étrangers, des laboratoires publics ou privés. 


\title{
New image reconstruction algorithm for fluorescence optical tomography based on the adjoint radiative transfer equation
}

\author{
Fatmir Asllanaj $j^{\mathrm{a}}$ and Ahmad Addoum ${ }^{\mathrm{a}}$ \\ ${ }^{a}$ University of Lorraine, ENSEM, LEMTA, F-54518, Nancy, France
}

\begin{abstract}
A new reconstruction algorithm for fluorescence optical tomography of biological tissues is proposed. The radiative transport equation in the frequency domain is used to model light propagation. The adjoint method studied in this work provides an efficient way for solving the inverse problem. The methodology is applied to a $2 \mathrm{D}$ tissue-like phantom subjected to a collimated laser beam. Indocyanine Green is used as fluorophore. Reconstructed images of the spatial fluorophore absorption distribution is assessed taking into account the residual fluorescence in the medium. We show that illuminating the tissue surface from a collimated centered direction near the inclusion gaves a better reconstruction quality. Two closely positioned inclusions can be accurately localized and quantified. However, the algorithm fails to reconstruct smaller or deeper inclusions due to light attenuation in the medium. Reconstructions with noisy data are also achieved with a reasonable accuracy.
\end{abstract}

Keywords: fluorescence molecular imaging, radiative transport equation, inverse source problem, Lagrangian optimization, cancer diagnosis.

\section{INTRODUCTION}

In recent years, fluorescence imaging has received particular attention. This is due considerable potential for biomedical research and clinical applications. ${ }^{1}$ The quantification of fluorophore absorption and its distribution through biological tissues is of major interest. Fluorescence Diffuse Optical Tomography (FDOT) is an imaging method that aims to reconstruct the internal distribution of fluorochromes or chromophores within biological tissues. This is based on light measurements collected at the tissue surface. ${ }^{1-3}$ Especially in small animals, this technology has facilitated monitoring of molecular activity, tumor growth, response to drug therapy, etc. In FDOT, a near-infrared excitation light source is used to measure fluorescence emission. Detection uses a CCD camera opposite the source that is rotated around the subject. However, due to the diffuse nature of light propagation in biological tissues, the image reconstruction problem is ill-posed and the images obtained are of low resolution. The reconstruction of the optical properties of the medium requires an accurate forward model for light propagation combined with an efficient inverse method.

The radiative transport equation (RTE) is considered as the gold standard for accurate prediction of light propagation through biological tissues at both the meso and the macroscale. I.e. the typical length of the scattering medium, beam diameter, etc. are high compared to the wavelength of the incident beam. ${ }^{3}$ Several studies deal with fluorescence molecular imaging based on the diffusion equation used as forward model. The diffusion equation is an approximation relative to the RTE and has limitations in optically thin media. Equally, it fails in media with small geometries where boundary effects are dominant and where sources and detectors are not sufficiently far apart. This presents specific problems for example in the field of small animal fluorescence imaging. Their fluorescent sources are potentially very close to detectors on the tissue surface. An image reconstruction method based on the RTE overcomes these limitations. There are very few reports in the literature concerning the inverse fluorescent source problem in fluorescence molecular imaging based on RTE as the forward model. Accordingly, this problem needs further investigation.

Further author information: (Send correspondence to Fatmir Asllanaj)

E-mail: Fatmir.asllanaj@univ-lorraine.fr,

Telephone: +33 (0) 372744273 
The image reconstruction methods based on the RTE can be found $i^{4}$ (steady-state domain) and ${ }^{5,6}$ (frequency domain). There was also considerable work on developing other RTE-based methods for optical imaging. ${ }^{7,12}$ The fluorescent source reconstruction is achieved by minimizing an objective function. This latter describes the discrepancy between measured light intensity and predicted numerical data on the tissue surface. The gradient of the objective function is a crucial indication of update through line minimization of the unknown fluorescent source distribution. The adjoint method is known to be efficient for inverse problems. It is used in research areas covered by shape optimization, fluid flow control, etc. The adjoint equation is derived from its primal equation using integration by parts. Gradient values can be efficiently calculated from a particular quantity of interest by solving the adjoint equation. The computation of the objective function gradient for large-scale problems using the finite differences method is extremely time consuming. A distinct advantage of using the adjoint method is that an explicit expression of the gradient is obtained that allows the gradient to be computed efficiently. This is achieved by solving an additional (adjoint) equation for the adjoint variable. The computational cost is equivalent to that of the forward model.Furthermore, the adjoint method is independent from the number of parameters. Thus, it allows more accurate computing of the objective function gradient and the computational cost is much lower than for the finite differences method. The adjoint method was applied to DOT problems based on RTE in the time domain ${ }^{8}$ and the frequency domain. ${ }^{9}$ In the reports cited, a forward model including partly reflected boundary conditions (Fresnel reflection) was considered in. ${ }^{9}$ The adjoint method was applied to FDOT problems based on the RTE frequency domain in. ${ }^{5,6} \mathrm{In},{ }^{5}$ a unit strength isotropic source located at one point and transparent boundary conditions were considered in the forward model. In, ${ }^{6}$ a unit strength isotropic source located at four points with partly reflected boundary conditions was considered in the forward model. The Lagrangian formulation and the forward model were solved implicitly using a linear system. This paper proposes a reconstruction algorithm for FDOT using the RTE in the frequency domain to model the light propagation. The adjoint method studied in this work provides an efficient way for solving the inverse problem of FDOT based on RTE.

The remainder of this manuscript is organized as follows. Section 2 presents the forward model in a 2D medium, subjected to a collimated laser beam. Section 3 deals with the mathematical background of the Lagrangian formulation to derive the adjoint RTE and the objective function gradient. Section 4 discusses the results of the fluorophore absorption coefficient reconstructions with simulated data. Conclusions are summarized in the final section.

\section{FORWARD MODEL}

\subsection{Excitation light propagation}

The $\psi_{c}^{e x}(\boldsymbol{r}, \omega)$ collimated excitation radiance at location $\boldsymbol{r} \in \mathcal{D}$ in direction $\boldsymbol{\Omega}_{\boldsymbol{c}}$ and angular modulation frequency $\omega \in \mathbb{R}$ is governed by the Bouguer-Beer-Lambert attenuation equation:

$$
\left[\boldsymbol{\Omega}_{c} \cdot \nabla+\left(\frac{i \omega}{v^{e x}}+\mu_{t}^{e x}(\boldsymbol{r})+\mu_{a}^{e x \rightarrow e m}(\boldsymbol{r})\right)\right] \psi_{c}^{e x}(\boldsymbol{r}, \omega)=0 \text { for } \boldsymbol{r} \in \mathcal{D}
$$

The $\mu_{a}^{e x \rightarrow e m}$ coefficient is the absorption coefficient of a fluorescent source in the tissue at the excitation wavelength $\lambda^{e x}$. The speed of light $v^{e x}$ in the tissue, is given by the ratio $v^{e x}=c / n^{e x}$ of the speed of light in vacuum and the refractive index of the tissue. The boundary conditions of Eq. 1 are:

$$
\psi_{c}^{e x}(\boldsymbol{r}, \omega)-\Upsilon^{e x}(\boldsymbol{r}, \omega)=0 \text { for } \boldsymbol{r} \in \partial \mathcal{D}_{c} \text { and } \psi_{c}^{e x}(\boldsymbol{r}, \omega)=0 \text { for } \boldsymbol{r} \in \partial \mathcal{D} \backslash \partial \mathcal{D}_{c}
$$

The diffuse excitation radiance $\psi_{s}^{e x}(\boldsymbol{r}, \boldsymbol{\Omega}, \omega)$ in direction $\Omega$ is the solution of the RTE in the frequency domain at an excitation wavelength of $\lambda^{e x}$ :

$$
\begin{gathered}
{\left[\boldsymbol{\Omega} \cdot \nabla+\left(\frac{i \omega}{v^{e x}}+\mu_{t}^{e x}(\boldsymbol{r})+\mu_{a}^{e x \rightarrow e m}(\boldsymbol{r})\right)\right] \psi_{s}^{e x}(\boldsymbol{r}, \boldsymbol{\Omega}, \omega)} \\
-\mu_{s}^{e x}(\boldsymbol{r}) \int_{\Omega^{\prime}=2 \pi} p^{e x}\left(\boldsymbol{\Omega}^{\prime} \cdot \boldsymbol{\Omega}\right) \psi_{s}^{e x}\left(\boldsymbol{r}, \boldsymbol{\Omega}^{\prime}, \omega\right) d \Omega^{\prime}-S_{c}^{e x}(\boldsymbol{r}, \boldsymbol{\Omega}, \omega)=0,
\end{gathered}
$$


where $S_{c}^{e x}(\boldsymbol{r}, \boldsymbol{\Omega}, \omega)=\mu_{s}^{e x}(\boldsymbol{r}) p^{e x}\left(\boldsymbol{\Omega}_{\boldsymbol{c}} \cdot \boldsymbol{\Omega}\right) \psi_{c}^{e x}(\boldsymbol{r}, \omega)$ is a source term and $p^{e x}\left(\boldsymbol{\Omega}^{\prime} \cdot \boldsymbol{\Omega}\right)$ is the Henyey-Greenstein (H-G) phase function. ${ }^{10}$ The boundary conditions for the diffuse excitation radiance are:

$$
\begin{gathered}
\psi_{s}^{e x}(\boldsymbol{r}, \boldsymbol{\Omega}, \omega)-\rho\left(\Theta_{s p}\right) \psi_{s}^{e x}\left(\boldsymbol{r}, \boldsymbol{\Omega}_{\boldsymbol{s p}}, \omega\right)=0 \text { with } \cos \Theta_{s p}=\boldsymbol{\Omega}_{\boldsymbol{s p}} \cdot \boldsymbol{n}=-\boldsymbol{\Omega} \cdot \boldsymbol{n} \text { (specular reflection), } \\
\psi_{s}^{e x}(\boldsymbol{r}, \boldsymbol{\Omega}, \omega)-\frac{1}{\pi} \int_{\boldsymbol{\Omega}^{\prime} \cdot \boldsymbol{n}>0} \rho\left(\Theta^{\prime}\right) \psi_{s}^{e x}\left(\boldsymbol{r}, \boldsymbol{\Omega}^{\prime}, \omega\right) \boldsymbol{\Omega}^{\prime} \cdot \boldsymbol{n} d \Omega^{\prime}=0 \text { with cos } \Theta^{\prime}=\boldsymbol{\Omega}^{\prime} \cdot \boldsymbol{n} \text { (diffuse reflection), }
\end{gathered}
$$

The directional reflection coefficient $\rho$ is given by Snell-Descartes laws assuming that the refractive index of the outside medium (air) is unity. The specular reflection $\boldsymbol{\Omega}_{\boldsymbol{s} \boldsymbol{p}}=\boldsymbol{\Omega}-2(\boldsymbol{\Omega} \cdot \boldsymbol{n}) \boldsymbol{n}$ is defined as the direction from which a laser beam must hit the surface. The excitation reflectance $R^{e x}$ is obtained from the photon boundary flux at each boundary point such as: ${ }^{10}$

$$
R^{e x}(\boldsymbol{r}, \omega)=\int_{\boldsymbol{\Omega} \cdot \boldsymbol{n}>0}[1-\rho(\Theta)] \psi_{s}^{e x}(\boldsymbol{r}, \boldsymbol{\Omega}, \omega) \boldsymbol{\Omega} \cdot \boldsymbol{n} d \Omega \text { with } \cos \Theta=\boldsymbol{\Omega} \cdot \boldsymbol{n},
$$

\subsection{Emission light propagation}

The quantum yield of the fluorochrome is denoted $\eta$ and $\tau$ denotes the local lifetime of the fluorescent source. The fluorescent source term is defined by: ${ }^{1}$

$$
Q^{e m}(\boldsymbol{r}, \omega)=\frac{1}{2 \pi} \frac{\eta \mu_{a}^{e x \rightarrow e m}(\boldsymbol{r})}{1-i \omega \tau(\boldsymbol{r})} \Phi^{e x}(\boldsymbol{r}, \omega) \text { for } \boldsymbol{r} \in \mathcal{D}
$$

The source emits isotropic light, since all directional information is lost after excitation. The equation that describes the transport of emitted light at the emission wavelength $\lambda^{e m}$ due to the fluorescent source in tissues is expressed as:

$$
\left[\boldsymbol{\Omega} \cdot \boldsymbol{\nabla}+\left(\frac{i \omega}{v^{e m}}+\mu_{t}^{e m}(\boldsymbol{r})\right)\right] \psi^{e m}(\boldsymbol{r}, \boldsymbol{\Omega}, \omega)-\mu_{s}^{e m}(\boldsymbol{r}) \int_{\Omega^{\prime}=2 \pi} p^{e m}\left(\boldsymbol{\Omega}^{\prime} \cdot \boldsymbol{\Omega}\right) \psi^{e m}\left(\boldsymbol{r}, \boldsymbol{\Omega}^{\prime}, \omega\right) d \Omega^{\prime}-Q^{e m}(\boldsymbol{r}, \omega)=0,
$$

where $\psi^{e m}(\boldsymbol{r}, \boldsymbol{\Omega}, \omega)$ denotes the emission radiance. The boundary conditions for the emission radiance are:

$$
\begin{gathered}
\psi^{e m}(\boldsymbol{r}, \boldsymbol{\Omega}, \omega)-\rho\left(\Theta_{s p}\right) \psi^{e m}\left(\boldsymbol{r}, \boldsymbol{\Omega}_{\boldsymbol{s} \boldsymbol{p}}, \omega\right)=0 \text { with } \cos \Theta_{s p}=\boldsymbol{\Omega}_{\boldsymbol{s} \boldsymbol{p}} \cdot \boldsymbol{n}=-\boldsymbol{\Omega} \cdot \boldsymbol{n} \text { (specular reflection), } \\
\psi^{e m}(\boldsymbol{r}, \boldsymbol{\Omega}, \omega)-\frac{1}{\pi} \int_{\boldsymbol{\Omega}^{\prime} \cdot \boldsymbol{n}>0} \rho\left(\Theta^{\prime}\right) \psi^{e m}\left(\boldsymbol{r}, \boldsymbol{\Omega}^{\prime}, \omega\right) \boldsymbol{\Omega}^{\prime} \cdot \boldsymbol{n} d \Omega^{\prime}=0 \text { with cos } \Theta^{\prime}=\boldsymbol{\Omega}^{\prime} \cdot \boldsymbol{n} \quad \text { (diffuse reflection), }
\end{gathered}
$$

The emission photon boundary flux (emission reflectance $R^{e m}$ ) is:

$$
R^{e m}(\boldsymbol{r}, \omega)=\int_{\boldsymbol{\Omega} \cdot \boldsymbol{n}>0}[1-\rho(\Theta)] \psi^{e m}(\boldsymbol{r}, \boldsymbol{\Omega}, \omega) \boldsymbol{\Omega} \cdot \boldsymbol{n} d \Omega \text { with } \cos \Theta=\boldsymbol{\Omega} \cdot \boldsymbol{n}, \text { for } \boldsymbol{r} \in \partial \mathcal{D}
$$

\section{INVERSE FLUORESCENT SOURCE PROBLEM}

To define more easier the state equation presented therafter, we denote by $\mathcal{R}_{c}^{e x}\left(\cdot, \psi_{c}^{e x}\right), \mathcal{R}_{s}^{e x}\left(\cdot, \psi_{c}^{e x}, \psi_{s}^{e x}\right)$ and $\mathcal{R}^{e m}\left(\cdot, \psi_{c}^{e x}, \psi_{s}^{e x}, \psi^{e m}\right)$ the equations (1), (3) and (7).

\subsection{The objective function}

The spatial distribution of the fluorophore absorption coefficient $\mu_{a}^{e x \rightarrow e m}$ is reconstructed by applying a nonlinear optimization technique to an objective function $J$ that is an explicit function of $\theta=\mu_{a}^{e x \rightarrow e m}$. It describes the discrepancy between the measured light intensity, $d_{o b s}^{e m}(\boldsymbol{r})$ and the predicted numerical data, $R^{e m}(\boldsymbol{r})$ (given from Eq. (9)) for positions $r \in \partial \mathcal{D}_{c}$ :

$$
J(\theta)=\frac{1}{2}\left\|R^{e m}(\theta)-d_{o b s}^{e m}\right\|_{B}^{2}
$$

The reconstruction algorithm consists of minimizing $J$ when the state equations are satisfied :

$$
\mathcal{R}_{c}^{e x}\left(\theta, \psi_{c}^{e x}\right)=0, \quad \mathcal{R}_{s}^{e x}\left(\theta, \psi_{c}^{e x}, \psi_{s}^{e x}\right)=0 \text { and } \mathcal{R}^{e m}\left(\theta, \psi_{c}^{e x}, \psi_{s}^{e x}, \psi^{e m}\right)=0
$$

The optimization algorithm chosen in this work requires the objective function gradient with respect to unknowns. To compute this gradient, the adjoint method is introduced as described in the following section. 


\subsection{The Lagrangian and adjoint method}

The Lagrangian is written in the $L^{2}$ space as: ${ }^{11}$

$$
\mathcal{L}\left(\theta, \psi_{c}^{e x}, \psi_{s}^{e x}, \psi^{e m}, \phi_{c}^{e x}, \phi_{s}^{e x}, \phi^{e m}\right)=\frac{1}{2} \|\left(H \psi^{e m}\right)-d_{o b s}^{e m}||^{2}+\left\langle\phi_{c}^{e x} \mid \mathcal{R}_{c}^{e x}\right\rangle+\left\langle\phi_{s}^{e x} \mid \mathcal{R}_{s}^{e x}\right\rangle+\left\langle\phi^{e m} \mid \mathcal{R}^{e m}\right\rangle
$$

where $\phi_{c}^{e x}, \phi_{s}^{e x}$ and $\phi^{e m}$ represent the adjoint variables associated to $\psi_{c}^{e x}, \psi_{s}^{e x}, \psi^{e m}$ in the medium. The fundamental remark is that if $\left(\psi_{c}^{e x}, \psi_{s}^{e x}, \psi^{e m}\right)$ is the solution of the state equation (11) for the $\theta$ parameter, then we have the identity:

$$
\mathcal{L}\left(\theta, \psi_{c}^{e x}(\theta), \psi_{s}^{e x}(\theta), \psi^{e m}(\theta), \phi_{c}^{e x}, \phi_{s}^{e x}, \phi^{e m}\right)=J(\theta), \quad \text { for all } \phi_{c}^{e x}, \phi_{s}^{e x}, \phi^{e m}
$$

By deriving this equation with respect to $\theta$ it yields :

$$
J^{\prime}(\theta) \delta \theta=\langle\nabla J(\theta) \mid \delta \theta\rangle=\frac{\partial \mathcal{L}\left(\theta, \psi_{c}^{e x}, \psi_{s}^{e x}, \psi^{e m}, \phi_{c}^{e x}, \phi_{s}^{e x}, \phi^{e m}\right)}{\partial \theta} \delta \theta
$$

Using the definition of the adjoint operator ${ }^{13}$ and the inner product properties, the adjoint variables are solutions of the following adjoint equations model :

$$
\begin{gathered}
\begin{array}{c}
{\left[\boldsymbol{\Omega} \cdot \boldsymbol{\nabla}+\left(\frac{-i \omega}{v^{e m}}+u_{t}^{e m}(\boldsymbol{r})\right)\right] \phi^{e m}(\boldsymbol{r},-\boldsymbol{\Omega}, \omega)=\mu_{s}^{e m}(\boldsymbol{r}) \int_{\Omega^{\prime}=2 \pi} p^{e m}\left(\boldsymbol{\Omega}^{\prime} \cdot(-\boldsymbol{\Omega})\right) \phi^{e m}\left(\boldsymbol{r}, \boldsymbol{\Omega}^{\prime}, \omega\right) d \Omega^{\prime},} \\
{\left[\boldsymbol{\Omega} \cdot \boldsymbol{\nabla}+\left(\frac{-i \omega}{v^{e x}}+\mu_{t}^{e x}(\boldsymbol{r})+\mu_{a}^{e x \rightarrow e m}(\boldsymbol{r})\right)\right] \phi_{s}^{e x}(\boldsymbol{r},-\boldsymbol{\Omega}, \omega)} \\
=\mu_{s}^{e x}(\boldsymbol{r}) \int_{\Omega^{\prime}=2 \pi} p^{e x}\left(\boldsymbol{\Omega}^{\prime} \cdot(-\boldsymbol{\Omega})\right) \phi_{s}^{e x}\left(\boldsymbol{r}, \boldsymbol{\Omega}^{\prime}, \omega\right) d \Omega^{\prime}+\frac{\eta \mu_{a}^{e x \rightarrow e m}(\boldsymbol{r})}{1+i \omega \tau(\boldsymbol{r})} \phi^{e m}(\boldsymbol{r},-\boldsymbol{\Omega}, \omega), \\
{\left[\boldsymbol{\Omega}_{c} \cdot \boldsymbol{\nabla}+\left(\frac{-i \omega}{v^{e x}}+\mu_{t}^{e x}(\boldsymbol{r})+\mu_{a}^{e x \rightarrow e m}(\boldsymbol{r})\right)\right] \phi_{c}^{e x}(\boldsymbol{r}, \omega)} \\
=\mu_{s}^{e x}(\boldsymbol{r}) \int_{\Omega^{\prime}=2 \pi} p^{e x}\left(\boldsymbol{\Omega}^{\prime} \cdot\left(-\boldsymbol{\Omega}_{\boldsymbol{c}}\right)\right) \phi_{s}^{e x}\left(\boldsymbol{r}, \boldsymbol{\Omega}^{\prime}, \omega\right) d \Omega^{\prime}+\frac{1}{2 \pi} \frac{\eta \mu_{a}^{e x \rightarrow e m}(\boldsymbol{r})}{1+i \omega \tau(\boldsymbol{r})} \int_{\Omega^{\prime}=2 \pi} \phi^{e m}\left(\boldsymbol{r}, \boldsymbol{\Omega}^{\prime}, \omega\right) d \Omega^{\prime}
\end{array}
\end{gathered}
$$

The adjoint boundary conditions in the case of diffuse reflection of the first two equations of system (14) are:

$$
\begin{gathered}
\phi^{e m}(\boldsymbol{r}, \boldsymbol{\Omega}, \omega)=\frac{1}{\pi} \int_{\boldsymbol{\Omega}^{\prime} \cdot \boldsymbol{n}<0} \rho\left(\Theta^{\prime}\right) \phi^{e m}\left(\boldsymbol{r}, \boldsymbol{\Omega}^{\prime}, \omega\right)\left|\boldsymbol{\Omega}^{\prime} \cdot \boldsymbol{n}\right| d \Omega^{\prime} \text { for, } \\
\phi^{e m}(\boldsymbol{r}, \boldsymbol{\Omega}, \omega)=-[1-\rho(\Theta)]\left(R^{e m}-d_{o b s}^{e m}\right)(\boldsymbol{r}, \omega)+\frac{1}{\pi} \int_{\boldsymbol{\Omega}^{\prime} \cdot \boldsymbol{n}<0} \rho\left(\Theta^{\prime}\right) \phi^{e m}\left(\boldsymbol{r}, \boldsymbol{\Omega}^{\prime}, \omega\right)\left|\boldsymbol{\Omega}^{\prime} \cdot \boldsymbol{n}\right| d \Omega^{\prime} \text { for, } \\
\phi_{s}^{e x}(\boldsymbol{r}, \boldsymbol{\Omega}, \omega)=\frac{1}{\pi} \int_{\boldsymbol{\Omega}^{\prime} \cdot \boldsymbol{n}<0} \rho\left(\Theta^{\prime}\right) \phi_{s}^{e x}\left(\boldsymbol{r}, \boldsymbol{\Omega}^{\prime}, \omega\right)\left|\boldsymbol{\Omega}^{\prime} \cdot \boldsymbol{n}\right| d \Omega^{\prime},
\end{gathered}
$$

with $\cos \Theta^{\prime}=-\boldsymbol{\Omega}^{\prime} \cdot \boldsymbol{n}$ and $\cos \Theta=\boldsymbol{\Omega} \cdot \boldsymbol{n}$. It can be seen that the adjoint equations model takes a similar form to the forward model. They can be solved in a similar manner to that used to solve the forward model. It also shows that the set of equations should be solved in the order presented in (14). The adjoint emission equation must be solved before the adjoint excitation equation. Thus, the emission adjoint field $\phi^{e m}$ obtained first, is used as the source term in the other two equations. After the first equation is solved, the second can be worked out and the excitation diffuse adjoint field $\phi_{s}^{e x}$ deduced. The resulting $\phi_{s}^{e x}$ field is used as the source term for the excitation collimated adjoint field $\phi_{c}^{e x}$.

\subsection{Gradient of the objective function and the optimization problem}

As the objective function does not depend explicitly on $\theta$ (see Eq. (10)), then we have $\frac{\partial J(\theta)}{\partial \theta}=0$. Using Eq. (13), we have :

$$
\langle\nabla J(\theta) \mid \delta \theta\rangle=\left\langle\phi_{c}^{e x} \mid \frac{\partial \mathcal{R}_{c}^{e x}}{\partial \theta} \delta \theta\right\rangle+\left\langle\phi_{s}^{e x} \mid \frac{\partial \mathcal{R}_{s}^{e x}}{\partial \theta} \delta \theta\right\rangle+\left\langle\phi^{e m} \mid \frac{\partial \mathcal{R}^{e m}}{\partial \theta} \delta \theta\right\rangle .
$$


Applying Eq. (18) to $\theta=\mu_{a}^{e x \rightarrow e m}$ we deduce that:

$$
\begin{aligned}
\left\langle\nabla J\left(\mu_{a}^{e x \rightarrow e m}\right) \mid \delta \mu_{a}^{e x \rightarrow e m}\right\rangle= & \left\langle\phi_{c}^{e x} \mid \psi_{c}^{e x} \delta \mu_{a}^{e x \rightarrow e m}\right\rangle+\left\langle\phi_{s}^{e x} \mid \psi_{s}^{e x} \delta \mu_{a}^{e x \rightarrow e m}\right\rangle \\
& -\left\langle\phi^{e m} \mid\left(\frac{1}{2 \pi} \frac{\eta}{1-i \omega \tau} \Phi^{e x}\right) \delta \mu_{a}^{e x \rightarrow e m}\right\rangle
\end{aligned}
$$

Then, the components of the objective function gradient, with respect to $\mu_{a}^{e x \rightarrow e m}$, are obtained with:

$$
\nabla J\left(\mu_{a}^{e x \rightarrow e m}\right)=\Re\left\{\phi_{c}^{e x} \overline{\psi_{c}^{e x}}+\left\langle\phi_{s}^{e x} \mid \overline{\psi_{s}^{e x}}\right\rangle_{A}-\frac{1}{2 \pi}\left\langle\phi^{e m} \mid \frac{\eta}{1+i \omega \tau} \overline{\Phi^{e x}}\right\rangle_{A}\right\} .
$$

where $\Re\{z\}$ is the real part of the complex number $z$. The right-hand side of (19) is a complex value but only its real part is taken for the gradient of the objective function to be a real value. The objective function $J$ was iteratively minimized using the quasi-Newton algorithm with L-BFGS (Limited-Memory Broyden-FletcherGoldfarb-Shanno). ${ }^{2}$ It iteratively updates an initial estimate of the fluorophore absorption coefficient along a search direction. Once the minimum is found, the final result is the distribution of the fluorophore absorption coefficient. The updating procedure can be formulated as detailed in. ${ }^{4}$

\section{RESULTS AND DISCUSSION}

We present numerical results obtained with the reconstruction algorithm developed in the theoretical section. The calculations were carried out with an Intel Xeon Processor E5-2643, 3.3GHz, 32GoRAM, 8 cores. This last uses Hyper-Threading and Intel $\mathrm{C}$ compiler. The computation time for a reconstruction is about 3 hours. The algorithm was used to localize and quantify one and two fluorescent inclusions embedded in a biological medium. The simulations were carried out in a $2 \mathrm{D}$ circular domain with a $2 \mathrm{~cm}$ radius. The center of the circle is defined as the origin. The chosen size mimics fluorescence tomographic problems that are typical for small animal imaging. The same unstructured triangular mesh composed of 2,129 nodal points and 4,128 triangular elements was used for the forward and inverse solutions. Each inclusion is a disk of $0.4 \mathrm{~cm}$ radius and its center is located at different positions. The intrinsic optical properties of the medium, namely $\mu_{a}^{e x}=\mu_{a}^{e m}=0.1 \mathrm{~cm}^{-1}$ and $\mu_{s}^{e x}=\mu_{s}^{e m}=100 \mathrm{~cm}^{-1}$ were identical at the excitation and emission wavelengths. The anisotropy factor of the H-G phase function $g$ was set to 0.9 . This corresponds to a highly forward-scattering medium. The optical properties are typical for biological tissues in the near-infrared spectral range. The refractive indices of the medium and the surrounding (air) were chosen as $n=1.4$ and $n_{\text {out }}=1.0$, respectively. The medium surface was assumed to be semi-transparent with specular Fresnel reflection at the interface. ICG (Indocyanine Green) ${ }^{3}$ is the most widely-used fluorophore probe for biomedical applications and was effectively used here. The quantum yield and lifetime of the probe were homogeneously distributed with $\eta=0.012$ and $\tau=0.52$ ns, respectively. We intended to reconstruct the fluorophore absorption coefficient. To this end we allowed determination of the relative ICG concentration in the medium. This process is known as the absorption-contrast mode in FDOT. The fluorescent inclusions represent heterogeneities in the $\mu_{a}^{e x \rightarrow e m}$ coefficient. ${ }^{3}$ The medium is not ideal, meaning that some ICG molecules remained in the homogeneous background medium leading to residual fluorescence. Therefore, the fluorescence signal detected on the surface had not only been caused by fluorescent inclusions. The fluorophore absorption coefficient in the background was assigned the value $\mu_{a}^{e x \rightarrow e m}=0.01 \mathrm{~cm}^{-1}$. This value was used as the initial estimate in the reconstruction algorithm for all cases described below. A collimated source using ten boundary nodal points (positions all around the disc) was used. These were equally spaced by about $0.1 \mathrm{~cm}$ and their centered points were denoted $\boldsymbol{P}_{\boldsymbol{c}}$. 64 detectors were used that where equally spaced along the boundary nodal points of reflectance. The intensity of the source was modulated at $100 \mathrm{MHz}$. The use of this modulation frequency leads to a large observation time which is longer than the fluorescence life time of ICG in order to obtain sufficient fluorescence decay. To solve the RTE, the angular space $(2 \pi \mathrm{Sr})$ was uniformly subdivided into 32 control solid angles. Furthermore, there were 8 subdivisions into a control solid angle for phase function normalization. ${ }^{10}$ The reconstruction was stopped when the normalized difference between two subsequent error functions was smaller than $10^{-5}$. We also tested other values for the initial estimate, namely $\mu_{a}^{e x \rightarrow e m}=10^{-5} \mathrm{~cm}^{-1}, \mu_{a}^{e x \rightarrow e m}=0.03 \mathrm{~cm}^{-1}$ and we obtained reconstruction results close to those for $\mu_{a}^{e x \rightarrow e m}=0.01 \mathrm{~cm}^{-1}$. In the first case, a fluorescent inclusion was embedded inside the homogeneous medium. Its center was located at the exact position $(1,1)$ as indicated by the dashed circle in Fig. 1 . The true fluorophore 
absorption coefficient in the inclusion was assigned $\mu_{a}^{e x \rightarrow e m}=0.05 \mathrm{~cm}^{-1}$. For all reconstructions, the synthetic fluorescence data were generated by running the forward model. The true spatial distribution of the fluorophore absorption coefficient we wanted to reconstruct in such a case was used. Particular attention was paid to the optimal configuration of source positions and their collimated directions. Figs. 1 and 2a depict the results when the collimated directions of the sources were $\boldsymbol{\Omega}_{\boldsymbol{c}}^{\mathbf{1}}=(0,-1)\left(\right.$ with $\left.\boldsymbol{P}_{\boldsymbol{c}}^{\mathbf{1}}=(0,2)\right)$ and $\boldsymbol{\Omega}_{\boldsymbol{c}}^{\mathbf{2}}=\left(\cos \left(\frac{5 \pi}{4}\right), \sin \left(\frac{5 \pi}{4}\right)\right)$ (with $\left.\boldsymbol{P}_{\boldsymbol{c}}^{\mathbf{2}}=\left(2 \cos \left(\frac{\pi}{4}\right), 2 \sin \left(\frac{\pi}{4}\right)\right)\right)$. The algorithm gave a well-defined spatial position of the fluorescent inclusion for both directions. However, the $\left(\boldsymbol{\Omega}_{\boldsymbol{c}}^{\mathbf{2}}, \boldsymbol{P}_{\boldsymbol{c}}^{\mathbf{2}}\right)$ source led to a superior reconstruction quality in terms of estimation and localization of the inclusion. With the $\left(\boldsymbol{\Omega}_{\boldsymbol{c}}^{\mathbf{1}}, \boldsymbol{P}_{\boldsymbol{c}}^{\mathbf{1}}\right)$ source, the inclusion was attached to the medium surface and was rather bean shaped. Absorption by the retrieved fluorophore in the inclusion was slightly underestimated relative to its real value. The surface near the inclusion could be illuminated with $\left(\boldsymbol{\Omega}_{\boldsymbol{c}}^{\mathbf{2}}, \boldsymbol{P}_{\boldsymbol{c}}^{\mathbf{2}}\right)$. Then, the circular shape and spatial position were clearly improved as was accuracy. Moreover, the local value retrieved in the inclusion was accurately estimated $\left(\mu_{a}^{e x \rightarrow e m}=0.05 \mathrm{~cm}^{-1}\right)$. We could then deduce the best configuration to accurately localize and quantify a fluorescent inclusion. It was to illuminate the medium surface near the inclusion using a centered collimated source. The reconstructed images of Fig. 1 and Fig. 2a were obtained after 129 and 138 iterations, respectively.

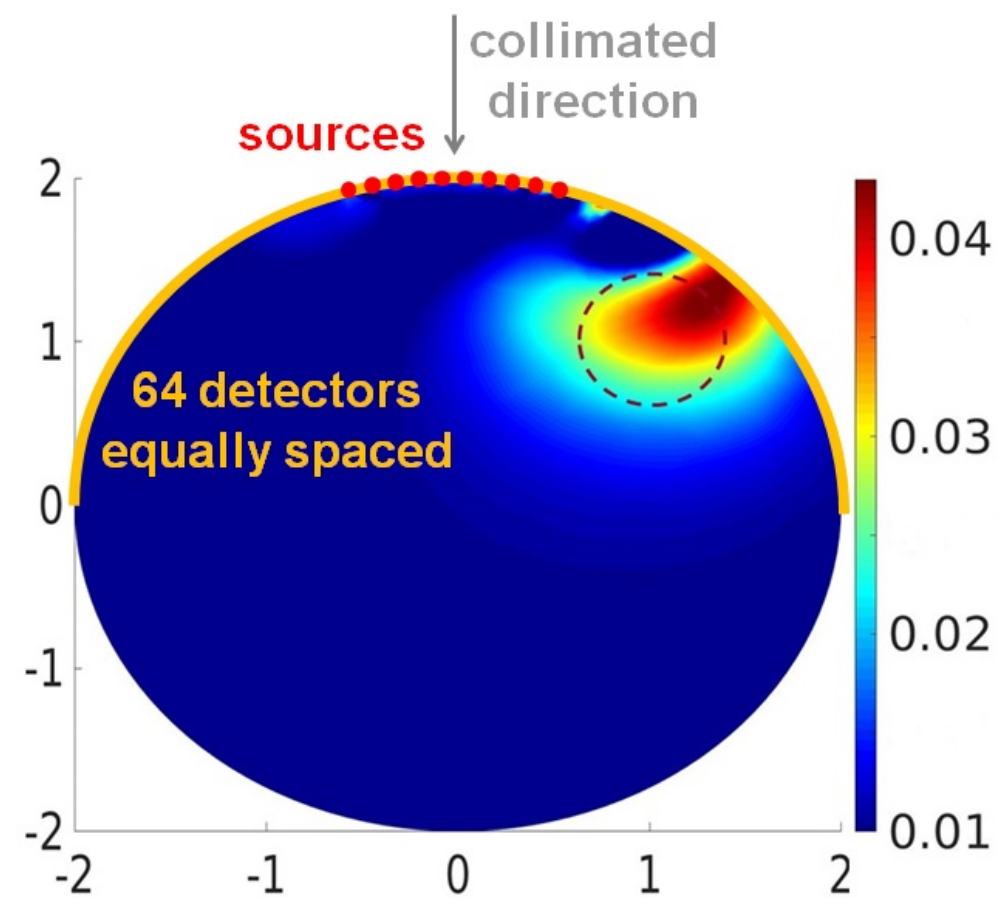

Figure 1: Reconstruction of the fluorescent inclusion. The center of the inclusion is located at $(1,1)$. The collimated direction of the source was $\boldsymbol{\Omega}_{\boldsymbol{c}}^{\mathbf{1}}=(0,-1)$ with $\boldsymbol{P}_{\boldsymbol{c}}^{\mathbf{1}}=(0,2)$.

In the second case we assessed the effect of the inclusion depth on the reconstruction. For this the center of the inclusion can take different exact positions, as shown by the dashed circle in Fig. 2. The $\boldsymbol{\Omega}_{\boldsymbol{c}}=\left(\cos \left(\frac{5 \pi}{4}\right), \sin \left(\frac{5 \pi}{4}\right)\right)$ direction (with $\boldsymbol{P}_{\boldsymbol{c}}=\left(2 \cos \left(\frac{\pi}{4}\right), 2 \sin \left(\frac{\pi}{4}\right)\right)$ ) was used as a collimated direction of the source. The estimation and spatial localization of the fluorescent inclusion became less accurate as the depth increased (Fig. 2). The algorithm failed to reconstruct deeper inclusions, leading to poor image quality. It should be noted that illuminating with more than one collimated sources didn't lead to improve the reconstruction. This could be explained by the ill-posedness of the whole problem, in particular light attenuation in the medium. The reconstructed image quality is assessed through the relative RMSE (Root Mean Square Error). This is defined between the true and 
reconstructed values of the fluorophore absorption coefficient:

$$
E=100 \cdot \frac{\| \mu_{a}^{e x \rightarrow e m}(\text { reconstructed })-\mu_{a}^{e x \rightarrow e m}(\text { true }) \|_{2}}{\| \mu_{a}^{e x \rightarrow e m}(\text { true }) \|_{2}},
$$

where $\|\cdot\|_{2}$ denotes Euclidian norm. The relative RMSE was computed over the target region and over the whole reconstructed image domain. As expected, Table 1 shows that the relative RMSE increases with the inclusion depth, much more for the target region than that for the whole reconstructed image domain.

\begin{tabular}{|c|c|c|c|c|}
\hline Position of the center of the inclusion & $(1,1)$ & $(0.8,0.8)$ & $(0.5,0.5)$ & $(0.0)$ \\
\hline Relative RMSE of the target region & $27.22 \%$ & $55.00 \%$ & $71.10 \%$ & $78.55 \%$ \\
\hline Relative RMSE of the whole image domain & $36.14 \%$ & $45.53 \%$ & $54.18 \%$ & $54.13 \%$ \\
\hline
\end{tabular}

Table 1: The relative RMSE versus the inclusion depth.

(a)

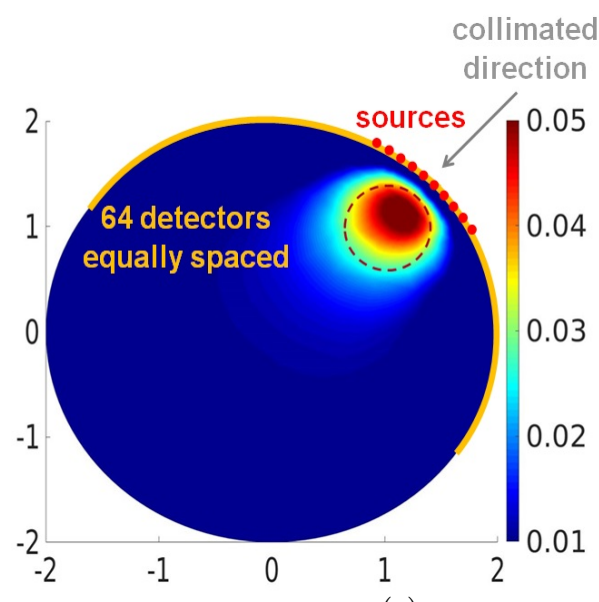

(c)

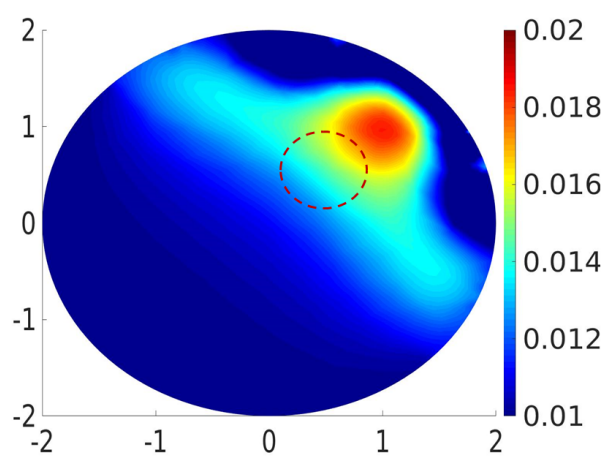

(b)

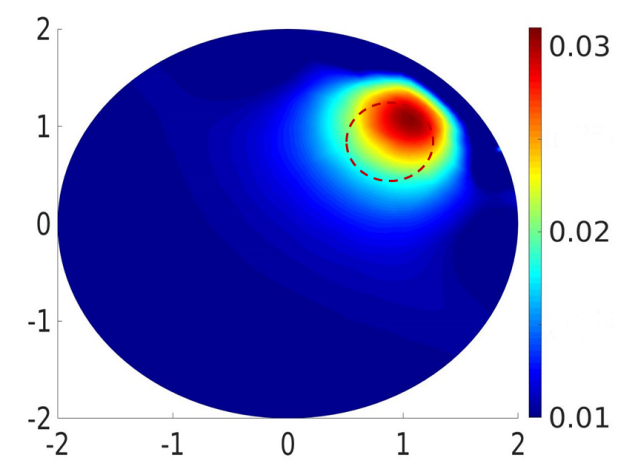

(d)

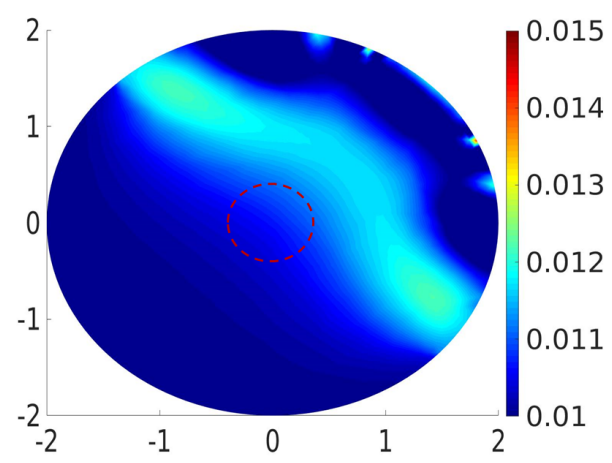

Figure 2: Reconstruction of the fluorescent inclusion located at different positions.

As a third case, we assessed the effect of both size and depth of the inclusion on the reconstruction. The relative RMSE over the target region is given in Fig. 3a. For each inclusion size, the error decreases as the center of the inclusion moves toward the sources positions. This is because the reconstruction (of shapes and values) is improved when the inclusion is located nearer to the illuminated medium boundary. At each position of the inclusion in the medium, the relative RMSE increases when the inclusion size decreases. As expected, the algorithm failed more to reconstruct a small inclusion located deeper in the medium. Fig. 3b shows the relative RMSE over the whole reconstructed image domain versus the depth and size of the inclusion. The error is lower (less than $36.5 \%$ ) when the inclusion size is smallest $(R=0.2 \mathrm{~cm})$ whatever its position in the medium. 
When the inclusion is deeper in the medium (located at the center of the medium) the relative RMSE is inverted compared to that over the target region. This means that the reconstruction quality of the whole domain is worst when the inclusion size is bigger and the inclusion is deeper in the medium. Moreover, when $R$ is bigger than $0.2 \mathrm{~cm}$, the relative RMSE over the whole domain increases as the inclusion goes deeper. This is mainly due to the wrong inclusion location inside the homogeneous medium which leads, therefore, to a poor reconstruction quality of the whole domain.

(a)

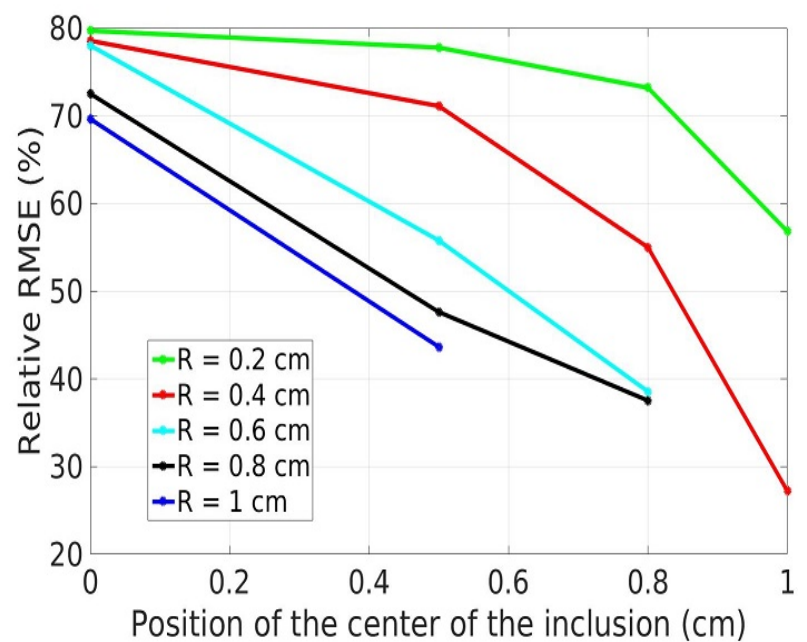

(b)

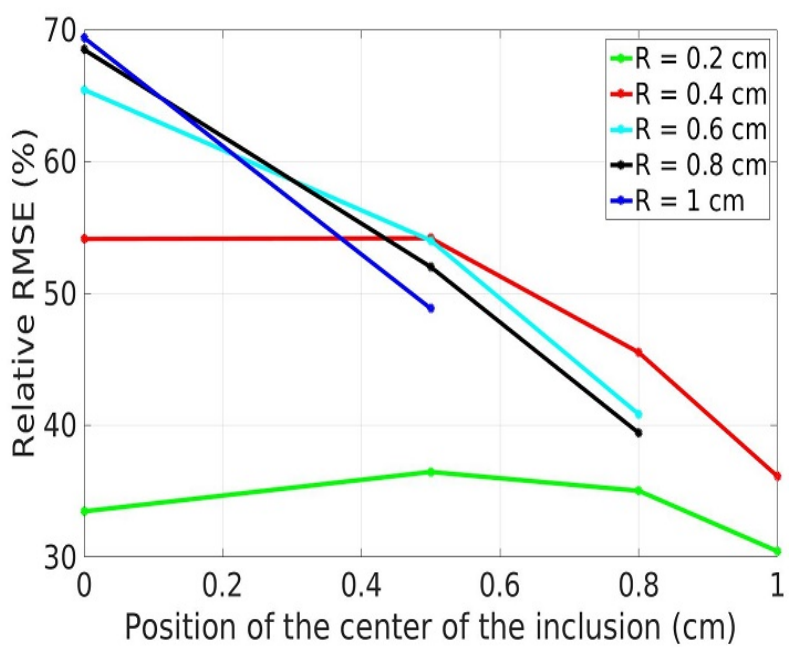

Figure 3: The relative RMSE versus the size and depth of the inclusion.

In the thourth case, a second inclusion with a lower fluorophore absorption coefficient $\left(\mu_{a}^{e x \rightarrow e m}=0.03 \mathrm{~cm}^{-1}\right)$ was considered. This allowed us to distinguish between two fluorescent inclusions at different separations. For this purpose, two examples are presented. Fig. 4a shows the reconstructed image when both inclusions were closed. The centers of the inclusions were located at $(-1,1)$ and $(1,0.8)$ and the phantom medium was simultaneously illuminated by $\boldsymbol{\Omega}_{\boldsymbol{c}}^{\boldsymbol{l}}=\left(\cos \left(\frac{7 \pi}{4}\right), \sin \left(\frac{7 \pi}{4}\right)\right)$ with $\boldsymbol{P}_{\boldsymbol{c}}^{\boldsymbol{l}}=\left(2 \cos \left(\frac{3 \pi}{4}\right), 2 \sin \left(\frac{3 \pi}{4}\right)\right)$ and $\boldsymbol{\Omega}_{\boldsymbol{c}}^{r}=\left(\cos \left(\frac{5 \pi}{4}\right), \sin \left(\frac{5 \pi}{4}\right)\right)$ with $P_{\boldsymbol{c}}^{\boldsymbol{r}}=\left(2 \cos \left(\frac{\pi}{4}-\frac{\pi}{32}\right), 2 \sin \left(\frac{\pi}{4}-\frac{\pi}{32}\right)\right)$. Fig. $4 \mathrm{~b}$ depicts the result for a somewhat higher separation between the two inclusions. Here, the centers of the inclusions were located at $(-1,1)$ and $(1.4,0)$ and the medium was illuminated by $\boldsymbol{\Omega}_{\boldsymbol{c}}^{\boldsymbol{l}}=\left(\cos \left(\frac{7 \pi}{4}\right), \sin \left(\frac{7 \pi}{4}\right)\right)$ with $\boldsymbol{P}_{\boldsymbol{c}}^{\boldsymbol{l}}=\left(2 \cos \left(\frac{3 \pi}{4}\right), 2 \sin \left(\frac{3 \pi}{4}\right)\right)$ and $\boldsymbol{\Omega}_{\boldsymbol{c}}^{\boldsymbol{r}}=(-1,0)$ with $\boldsymbol{P}_{\boldsymbol{c}}^{\boldsymbol{r}}=(2,0)$. The dashed circles in the figures indicate the exact positions of the inclusions. For both separations, the inclusions were accurately recovered and localized in the medium. The algorithm can detect and reconstruct separately the two different fluorescent inclusions. This is so even when they are only separated by small distances (see Fig. 4a). The mean retrieved values of both inclusions in the small and large separation cases are $\left(0.024 \mathrm{~cm}^{-1}\right.$, $\left.0.031 \mathrm{~cm}^{-1}\right)$ and $\left(0.025 \mathrm{~cm}^{-1}, 0.038 \mathrm{~cm}^{-1}\right)$, respectively. The mean retrieved values of inclusions were always underestimated compared to their exact values. Also, when the inclusions are relatively close to each other the fluorescent photons interfere.

In the last case study, the robustness of the reconstruction algorithm was tested in the presence of noisy data with Gaussian distribution. ${ }^{14}$ The noise was added to the complex simulated measurement reflectance (module and phase shift). The simulated measurement data are corrupted by adding random errors: $3 \%, 6 \%$ and 10 $\%$. Like the first case, the probed medium contains one inclusion. The relative RMSE versus the noise level is shown in Table 2.

\begin{tabular}{|c|c|c|c|c|}
\hline Noise & $\sigma_{m}=0 \%$ & $\sigma_{m}=3 \%$ & $\sigma_{m}=6 \%$ & $\sigma_{m}=10 \%$ \\
\hline Relative RMSE of the target region & $27.22 \%$ & $29.69 \%$ & $30.97 \%$ & $31.16 \%$ \\
\hline Relative RMSE of the reconstructed image domain & $36.14 \%$ & $39.58 \%$ & $49.31 \%$ & $63.35 \%$ \\
\hline
\end{tabular}

Table 2: The relative RMSE versus the noise level.

The example of $10 \%$ noise is illustrated in Fig. 5. This figure shows that despite the relatively high noise 
(a)

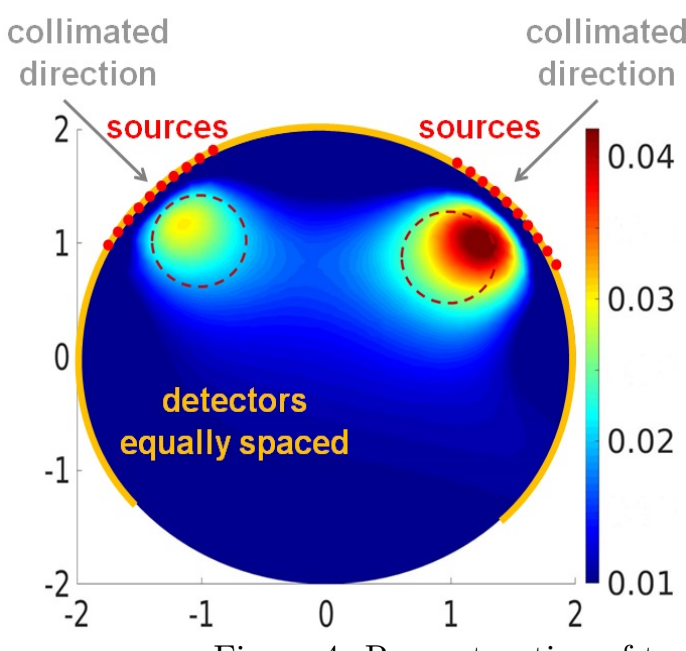

(b)

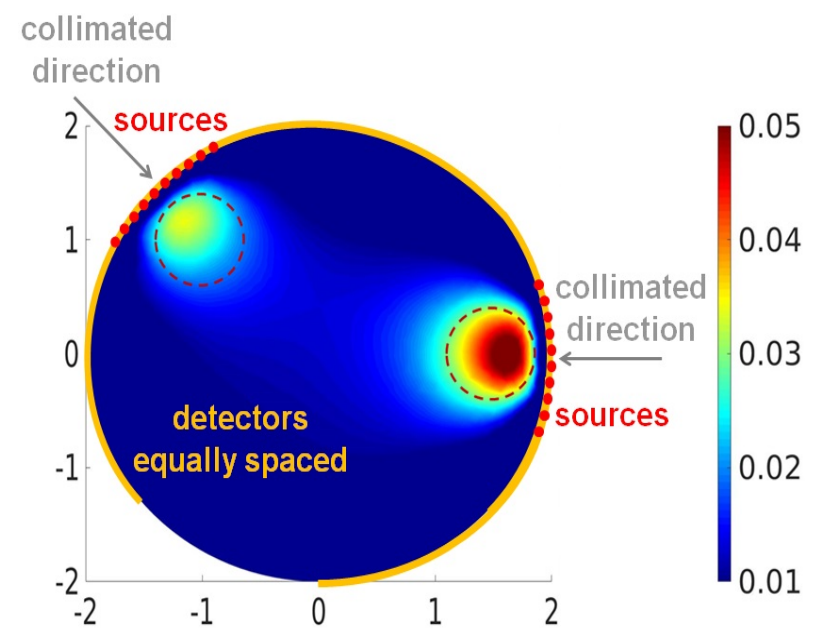

Figure 4: Reconstruction of two fluorescent inclusions located at different positions.

level, the inclusion is accurately reconstructed. Furthermore, the local retrieved value is well-estimated. This confirmed that the proposed algorithm is robust and efficient, even in the presence of noisy data. However, artifacts and some perturbations of the medium boundary are note compared to the noise-less reconstructed image (see Fig. 2a). This is because detection occurred on the surface and the algorithm converged around a random value. Higher noise levels for the boundary data led to degraded image quality. Relative RMSE decreases with the noise level, much more for the whole reconstructed image domain than that for the target region. (see Table 2).

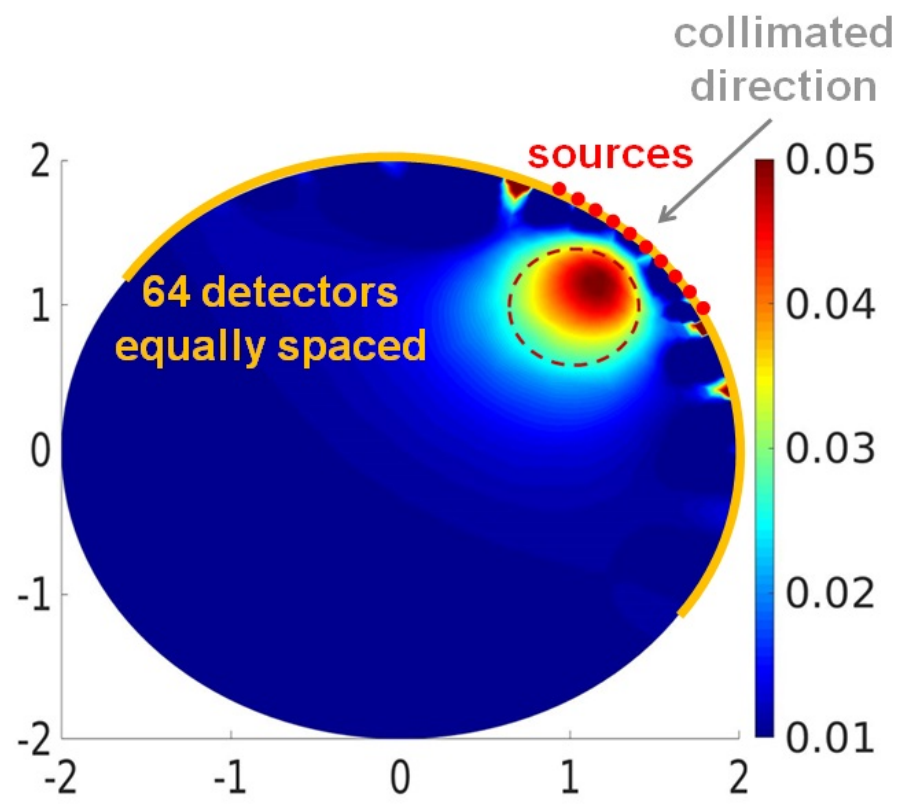

Figure 5: Reconstruction of the fluorescent inclusion when $10 \%$ of noise was added to the simulated measurement data. The center of the inclusion is located at $(1,1)$. 


\section{CONCLUSION}

We studied the inverse problem of ICG fluorescent source. For the first time, we derived the adjoint model for fluorescence molecular imaging based on the RTE to efficiently obtain the objective function gradient. This is ensured by solving an additional (adjoint) equation for the adjoint variable. The latters's computational cost is equivalent to that of the forward model. The coupled forward and adjoint models were solved with the same modified finite volume method. This had been demonstrated in a previous publication to be highly accurate. ${ }^{10}$ The objective function was iteratively minimized using a nonlinear optimization L-BFGS algorithm. Reconstructed images were obtained based on simulated data on the tissue surface. Thus, the spatial fluorophore absorption distribution was assessed taking into account the residual fluorescence in the medium. Positions and directions of the sources were measured on a single fluorescent inclusion. We showed that illuminating the tissue surface from a collimated centered direction near the inclusion gave a better reconstruction quality. Furthermore, we analyzed the effect of both size and depth of the inclusion on the reconstruction. We found that the algorithm failed to reconstruct smaller or deeper inclusions. This was due to light attenuation in the medium. Also, two closely positioned inclusions could be accurately localized. Additionally, their fluorophore absorption coefficients could be quantified. Reconstructions with noisy data were achieved with a reasonable accuracy for several random noise levels. The sum of our results demonstrated that the algorithm is robust. Also, it yields promising results in fluorescence molecular imaging. The present work was a prerequisite study to evaluate the potential of the algorithm. We plan to extend it to 3D geometries (for real applications) using parallel computing with MPI and Open MP, running on a set of multi-core machines.

\section{REFERENCES}

[1] Ntziachristos V 2006 Fluorescence molecular imaging Annu. Rev. Biomed. Eng. 8 1-33

[2] Arridge S R and Schotland J C 2009 Optical tomography: forward and inverse problems Inverse Problems 25123010

[3] Klose A D 2009 Light Scattering. Reviews 4, Book Chapter: Radiative transfer of luminescence light in biological tissue (Berlin Heidelberg: Springer:293-345)

[4] Klose A D, Ntziachristos V and Hielscher A H 2005 The inverse source problem based on the equation of radiative transfer in optical molecular imaging J. Comp. Phys. 202 323-345

[5] Joshi A, Rasmussen J C, Sevick-Muraca E M, Wareing T A, McGhee J 2008 Radiative Transport Based Frequency Domain Fluorescence Tomography Phys. Med. Biol. 53 2069-2088

[6] Kim H K, Lee J H and Hielscher A H 2010 PDE-constrained Fluorescence Tomography with the FrequencyDomain Equation of Radiative Transfer IEEE Journal of Selected Topics in Quantum Electronics 16

[7] Aydin E D 2007 Three-dimensional photon migration through void-like regions and channels Appl. Opt. 46 $8272-8277$

[8] Boulanger J, Liu F and Charette A 2007 Investigation on the reflection at the boundaries for reconstruction laser-based imaging J. Quant. Spectrosc. Radiat. Transfer 104 238-247

[9] Kim H K and Charette A 2007 A sensitivity function-based conjugate gradient method for optical tomography with the frequency-domain equation of radiative transfer J. Quant. Spectrosc. Radiat. Transfer 104 24-39

[10] Asllanaj F, Contassot-Vivier S, Liemert A and Kienle A 2014 Radiative transfer equation for predicting light propagation in biological media: comparison of a modified finite volume method, the Monte Carlo technique and an exact analytical solution J. Biomed. Opt. 19015002

[11] Bonnans J F, Gilbert JC, Lemarechal C and Sagastizabal C A 2016 Numerical Optimization Theoretical and Practical Aspects. (Springer Sciences and Business Media)

[12] Asllanaj F, Addoum A and Contassot-Vivier S 2015 Detection of tumor-like inclusions embedded within human liver tissue using a short-pulsed near-infrared laser beam : parallel simulations with radiative transfer equation J. Quant. Spectrosc. Radiat. Transfer 165 1-11

[13] Kern M 2011 Problmes inverses : aspects numriques (Hal archives-ouvertes.fr)

[14] Qiao Y, Qi H, Chen Q, Ruan L and Tan H 2015 Multi-start iterative reconstruction of the radiative parameter distributions in participating media based on the transient radiative transfer equation Opt. Comm. $35175-84$ 